\title{
Les agents virtuels intelligents : Quels atouts pour la relation client ?
}

\author{
Catherine Viot \\ Maître de Conférences - IAE de Bordeaux - Equipe de recherche en marketing de \\ l'IRGO (Université Bordeaux IV) \\ 35 avenue Abadie \\ 33072 Bordeaux Cedex \\ 0556009705 \\ viot33@free.fr \\ Chercheur associé à la chaire e-Commerce et Distribution de BEM - Bordeaux \\ Management School

\section{Grégory Bressolles} \\ Professeur de Marketing \\ Responsable de la chaire e-Commerce et Distribution \\ BEM - Bordeaux Ecole de Management \\ 680 Cours de la Libération \\ 33405 Talence \\ 0556842228 \\ gregory.bressolles@bem.edu
}

Les auteurs remercient les deux rédacteurs en chef ainsi que les trois relecteurs pour la qualité et la pertinence de leurs remarques, lesquelles ont permis d'améliorer cet article au fil des révisions. Leila Boutaleb, Marketing Manager de VirtuOz, est également remerciée pour sa disponibilité et les informations transmises.

Viot C., Bressolles G. (2012). Les agents virtuels intelligents : Quels atouts pour la relation client ? Décisions Marketing, 2012, N 65, pp.45-56. 


\title{
Les agents virtuels intelligents : quels atouts pour la relation client ?
}

\begin{abstract}
Résumé
Alors que les taux de conversion sur les sites marchands sont encore considérés comme faibles, que l'expérience d'achat en ligne est souvent perçue comme manquant de chaleur humaine et que les services consommateurs croulent sous les demandes d'informations et les réclamations, une solution existe. Elle consiste à intégrer un Agent Virtuel Intelligent (AVI) aux autres canaux de la relation client. Un bilan critique de la performance des AVI est d'abord effectué. Ces agents présentent des qualités liées aux progrès technologiques mais restent cependant perfectibles. Compte tenu de ce bilan, les atouts des AVI en matière de relation client - notamment leur influence sur les réponses des consommateurs, leur rôle dans l'humanisation du site et l'amélioration de la qualité de service, leur intégration dans le dispositif multicanal et la maîtrise des coûts opérationnels de relation client - sont ensuite discutés.
\end{abstract}

\section{Mots-clés}

Agent Virtuel Intelligent, marketing relationnel, relation client, Internet, présence sociale, qualité de service

\section{Intelligent Virtual Agents: What are the advantages for customer relationship?}

\begin{abstract}
While conversion rates on retail websites are still considered as low and while the customer experience of online purchase is often perceived as lacking human warmth and while consumer services collapse under information and complaints' requests, a solution exists. It is based on the integration of a Virtual Intelligent Agent (VIA) into the other customer relationship channels. A critical assessment of Virtual Intelligent Agents' performance is first made. They present qualities bound to the technological progress but they remain perfectible. Considering this assessment, the assets of the VIA in terms of customer relationship - in particular their influence on consumer outcomes, their role in the humanization of the website and the improvement of service quality, their integration into the multichannel customer relationship system and the control of operational costs - are then discussed.
\end{abstract}

\section{Key words}

Intelligent Virtual Agent, relationship marketing, customer relationship, Internet, social presence, service quality 
L'adoption d'Agents Virtuels Intelligents (AVI) par les sites Internet marchands s'est largement développée au cours de ces dernières années ; en témoigne l'élection de «Miss client 2010 et 2011 » réalisée par le site sensduclient.com ${ }^{1}$. Les AVI sont présentés comme un remède aux faibles taux de conversion (moins de $2 \%$ selon la FEVAD). Cette faible performance des sites trouve une première explication dans le manque de chaleur humaine ou de présence sociale ressentie par le prospect sur un site marchand, comparativement à un achat en magasin (4), mais aussi dans une assistance insuffisante, en temps réel, lors de l'achat. En effet, une étude du cabinet Forrester², réalisée en décembre 2009, auprès de plus de 4600 acheteurs en ligne nord américains, révèle que l'absence de réponse rapide à leur question inciterait $57 \%$ d'entre eux à mettre fin au processus d'achat.

Les AVI sont des représentations digitales de programmes informatiques qui ont été conçus pour interagir avec, ou se comporter comme un humain (11). Ils doivent être distingués des avatars qui constituent une représentation digitale des utilisateurs dans les univers virtuels. Alors que l'avatar n'est qu'une enveloppe contrôlée par un être humain, les AVI sont contrôlés par un ordinateur. Leur multiplication relève d'un phénomène plus général, celui du web self-service qui consiste à laisser le client ou le prospect être maître de sa relation avec l'entreprise en lui offrant, sur un mode intuitif et structuré, l'accès à l'ensemble des informations dont il peut avoir besoin, depuis le site web de l'entreprise. L'expression web self-care, issue d'une analogie avec le domaine médical où il est parfois demandé au patient de se prodiguer lui-même certains soins, est quelquefois employée par les praticiens. Ces deux expressions sont bien souvent utilisées de manière interchangeable. Le web self-service présente un intérêt pour l'entreprise qui met tout en œuvre pour que le prospect ou client trouve rapidement une réponse à sa question, directement sur le site Internet, sans avoir à entrer en contact par le canal téléphonique ou celui du courriel qui restent des points d'entrée plus coûteux de la relation client. En effet, selon la société VirtuOz, le coût d'un contact de relation client est estimé de 3 à 8 euros par email et de 8 à 20 euros par appel téléphonique et seulement de 20 centimes à 1 euro par conversation réussie par un AVI. La mise en place d'un AVI sur le site de la FNAC a permis à l'entreprise de réduire ses coûts opérationnels de $27 \%$. Pour Voyages-Sncf, le nombre d'emails entrants a été réduit de plus de 3000 par mois. Outre son intérêt économique pour l'entreprise, le web self- 
service est recherché par les clients. Selon l'étude de Forrester précédemment citée, 36 $\%$ des acheteurs en ligne préfèrent trouver une réponse sur le site Internet plutôt que d'entrer en contact par téléphone ou par email. Ce taux est même de $46 \%$ chez les 18 29 ans. Les AVI peuvent ainsi représenter un atout pour les entreprises engagées dans une logique relationnelle avec leurs clients car ils viennent en aide aux clients et prospects noyés par la masse d'informations disponibles sur les sites Internet, et améliorent également l'écoute du client. Cette technologie s'inscrit dans le courant du marketing relationnel.

Pour bon nombre de personnes, l'intelligence artificielle demeure un mythe plus qu'une réalité. Il n'est donc pas surprenant que les agents virtuels soient encore largement perçus comme de simples interfaces graphiques dépourvues d'intelligence. Au mieux, ils sont associés à un système de foires aux questions dynamiques (FAQ) doublé d'un moteur de recherche et représentés par un agent anthropomorphique.

Dans une première partie, un bilan critique de la performance des AVI est proposé. Celui-ci s'intéresse à l'intelligence des AVI et à leur aptitude à satisfaire aux missions pour lesquelles ils ont été implantés sur les sites Internet qui les accueillent. Compte tenu de ce bilan, les atouts des AVI relatifs à la relation client sont ensuite analysés en ce qui concerne leur influence sur les réponses du consommateur, leur potentiel d'humanisation du site et d'amélioration de la qualité de service, leur intégration dans le dispositif multicanal de la relation client et la maitrise des coûts opérationnels de celleci.

\section{La performance des AVI : un bilan critique}

Ce bilan sur les performances des AVI s'appuie sur plusieurs éléments : une revue de la littérature, notamment celle publiée par les chercheurs en systèmes d'information, des informations communiquées par les professionnels, et plus précisément par la Marketing Manager de la société VirtuOz (encadré 1) et une analyse de quatre agents, correspondant à quatre cas clients de VirtuOz: Clara (fnac.com), Lucie (assistance.sfr.fr), Nicolas Boulanger (finaref.fr) et Tea (discounteo.com). 


\section{Encadré 1 - La société VirtuOz}

VirtuOz a été créée en 2002 par trois ingénieurs linguistes et experts en intelligence artificielle. Après deux ans de mise au point des solutions, en collaboration avec le CNRS, les premières applications commerciales voient le jour et un premier client décisif adopte un AVI développé par l'entreprise en 2005 : il s'agit de Léa, qui guide les voyageurs au sein du site Voyages-Sncf. ViruOz connaît ensuite une véritable success story avec un chiffre d'affaires en progression de $60 \%$ entre 2007 et 2009 et le transfert de son siège social à San Francisco pour soutenir sa croissance outre Atlantique.

Le métier de VirtuOz n'est pas celui de l'animation de personnages, «c'est le cerveau humain », autrement dit, l'intelligence artificielle. Le discours du client est analysé sémantiquement. "Cela évite les contresens propres aux mots-clés : dites à un moteur de recherche 'je veux partir en voyage mais pas à la Martinique', il s'empressera de vous remonter les informations touristiques sur la Martinique $»^{3}$.

VirtuOz est parmi les leaders mondiaux avec des clients nationaux (Voyages-Sncf, MMA, Fnac.com, Crédit Agricole, Caisse Nationale d'Assurance Maladie, etc.) et des clients mondialement connus (eBay et PayPal, Nespresso, Coca Cola, par exemple).

Le business model est celui d'une licence et d'une facturation à la performance. Outre les frais de mise en place ${ }^{4}$, VirtuOz facture au client sous licence chaque « conversation réussie » de 20 centimes à 1 euro. Une conversation est qualifiée de réussie lorsque la mission confiée à l'agent a été correctement remplie. Les objectifs précis sont définis en partenariat avec le client. 80 à $90 \%$ des demandes traitées par un AVI sont qualifiées de conversations réussies. En général, 6 à 7 échanges sont nécessaires avant d'aboutir à une réponse définitive. Grâce à un Intranet, le client et VirtuOz ont accès, en temps réel, aux conversations et aux rapports d'analyse. La partie analytique permet d'évaluer le trafic et d'analyser les conversations. Elle fournit également des indicateurs permettant de mesurer la performance. L'AVI peut en outre être intégré au web analytic classique.

\section{Des atouts non négligeables}

Les AVI présentent des atouts non négligeables car ils peuvent être dotés d'un certain nombre d'attributs qui, jusque là, étaient réservés aux personnes humaines, comme la voix, l'écriture et l'intelligence. Ils peuvent ainsi se voir confier des missions précises comme l'aide à la navigation, l'aide avant-vente sur les sites riches en information, l'aide à l'achat et au SAV (service après-vente).

\section{- Les AVI : des agents intelligents ?}

Les AVI sont dotés de caractéristiques qui les font ressembler, à certains égards, aux êtres humains, mais disposent également de qualités qui leur sont propres.

Des agents aux qualités humaines de plus en plus sophistiquées. Les progrès technologiques ont rendu possible la création d'agents virtuels dotés d'attributs 
humains. En effet, ils sont non seulement capables d'écrire et de parler, mais ils comprennent également les clients et/ou les prospects qui s'adressent à eux en langage naturel. On pourrait penser qu'un AVI se contente de prononcer ou d'écrire des phrases stockées dans une base de données, en réponse à une requête comportant un certain nombre de mots-clés, sans pouvoir lui-même raisonner. Ce stade est aujourd'hui dépassé. Comme leur nom l'indique, les AVI disposent d'une intelligence artificielle. Dans les années 1950, Turing prédisait que d'ici l'an 2000 l'intelligence des ordinateurs rivaliserait avec celle des humains. Il avait d'ailleurs proposé un test permettant de confronter l'homme à la machine (19). Cette intelligence permet aux AVI de comprendre le sens contenu dans les questions formulées par les clients, grâce à l'analyse sémantique et d'y répondre en langage naturel. De manière plus générale, un agent intelligent est un programme qui est capable d'actions indépendantes dans un environnement donné, pour le compte d'un utilisateur. C'est un système qui perçoit son environnement et est capable d'agir sur celui-ci. En outre, l'AVI gagne en intelligence avec l'expérience. Une équipe de chercheurs taïwanais a développé un agent, dénommé ISA - pour Intelligent Sale Agent - capable d'argumenter, de faire des contreargumentations et de négocier un prix (5). Plusieurs expériences ont été conduites en laboratoire puis sur un site Internet spécialisé dans la vente de voitures d'occasion. L'expérience en ligne a permis de valider un effet positif de l'AVI sur la valeur perçue du produit, le consentement à payer et la satisfaction à l'égard du site.

Des agents qui peuvent s'affranchir des défauts de l'être humain. L'AVI présente des avantages par rapport aux êtres humains. En premier lieu, il n'est jamais fatigué ni malade. En conséquence, il est disponible pour les clients et prospects $24 \mathrm{~h} / 24$ et 7 jours/7 sans que cela n'ait d'influence sur son humeur. En outre, L'AVI ne perd pas patience et garde son calme face à un client désagréable, voire agressif, et il est suffisamment intelligent pour pouvoir déceler l'énervement d'un client et le basculer vers un autre canal de la relation client, grâce à un système de call-back, par exemple. L'AVI emploiera, en toutes circonstances, le ton et le vocabulaire idoine. Il n'aura pas recours aux négations et n'utilisera qu'un vocabulaire chargé d'un sens positif. Il évitera le jargon technique. Un autre avantage de l'AVI est qu'il sait quand il n'est pas en mesure de répondre. Au lieu de faire croire au client qu'il est lui-même à l'origine de 
son problème, comme le font parfois les opérateurs de hotlines, il reconnaîtra qu'il n'a pas la réponse et basculera le client vers un autre canal, en fonction du système d'escalade prévu (système de call-back, numéro de téléphone, etc.). Une autre qualité de l'agent intelligent est qu'il peut être polyglotte. Anna d'Ikea ou Louise d'eBay maîtrisent plusieurs langues. Il ne «raccrochera pas virtuellement au nez d'un interlocuteur qui s'adresserait à lui en anglais comme c'est encore malheureusement souvent le cas $\left(11 \%\right.$ des entreprises françaises $\left.{ }^{5}\right)$. Enfin, l'agent virtuel présente l'avantage de ne pas vieillir, contrairement aux êtres humains. Il restera ainsi toujours aussi attractif.

Des agents aux qualités « surhumaines ». Les AVI ne se contentent pas de copier les qualités des êtres humains. Par certains côtés, il faut bien reconnaître qu'ils peuvent les surpasser. En premier lieu, les AVI sont doués d'un don d'ubiquité qui leur permet de s'occuper simultanément de plusieurs clients. En effet, selon VirtuOz, un agent virtuel peut théoriquement traiter jusqu'à 4500 conversations simultanées (10 millions par mois), une caractéristique qui les distingue définitivement de l'être humain. Cette qualité des AVI permet de raccourcir le temps de réponse au client. L'AVI d'eBay traite 50 millions de conversations par an aux Etats-Unis.

L'AVI offre une grande souplesse en matière d'apparence physique. En effet, et si cela est souhaitable pour l'image ou l'identité de la marque, celui-ci peut revêtir exactement la même apparence physique pour tous les clients, qu'ils soient européens, américains, ou de tout autre pays dans lequel la marque est implantée. Par exemple, l'AVI d'eBay a toujours la même apparence ; en revanche, son prénom est adapté en fonction du pays (Louise en France, et Marie en Allemagne). Par ailleurs, d'un point de vue technique, il est tout à fait envisageable de pouvoir personnaliser l'apparence de l'AVI en fonction de différents critères (contextuels, choix de l'internaute, de l'entreprise...). Par exemple, Anna, l'AVI d'Ikea, est blonde en Allemagne, mais brune dans les autres pays. Cette possibilité peut contribuer à créer un sentiment d'empathie, facilitant l'identification à l'agent. Un consommateur asiatique préfèrera peut-être converser avec un agent de type asiatique.

Le profil de l'agent virtuel est défini en fonction d'un cahier des charges proposé par la marque. L'observation révèle que la plupart des AVI sont des femmes, excepté dans le secteur de la finance où les AVI masculins sont plus nombreux (Finaref, Crédit 
Agricole, Sofinco). La configuration de l'AVI ne se limite pas à son physique. On peut imaginer pouvoir aussi lui attribuer une personnalité bien spécifique, congruente avec la personnalité souhaitée pour la marque. Il est vrai qu'une entreprise peut recruter des collaborateurs disposant d'un profil cohérent avec l'image de l'entreprise mais cela peut s'avérer bien plus délicat que de «formater » un assistant virtuel.

\section{- Les AVI : des agents polyvalents}

Quelles sont les tâches confiées aux AVI ? L'entretien réalisé auprès de la Marketing Manager de VirtuOz a permis d'identifier les missions principales des AVI. A l'issu de cet entretien, nous avons obtenu l'autorisation d'analyser, plus en détail, quatre cas clients de la société (encadré 2).

\section{Encadré 2 - L'étude qualitative}

Quatre sites ayant adoptés un AVI développé par VirtuOz ont été analysés afin de déterminer le profil, la mission, les objectifs ainsi que les indicateurs de performance. Ces éléments d'observation ont été croisés avec les données disponibles sur le site de VirtuOz (études de cas, communiqués de presse) et les données collectées lors d'un entretien de 2 heures avec la Marketing Manager de VirtuOz. Les sites ont été sélectionnés en fonction de la diversité des secteurs d'activités (produits culturels et loisirs, produits financiers, téléphonie mobile, électroménager), de la diversité des agents (hommes/femmes ; photo/vidéo/dessin) et de la diversité des missions de l'AVI. Les autres AVI que nous avons étudiés n'offraient pas de missions différentes de celles de quatre agents retenus pour l'analyse.

Selon VirtuOz, quatre types de missions, correspondant à des étapes distinctes du processus d'achat, peuvent être confiées aux AVI (tableau 1). La première mission est une aide à la navigation particulièrement utile sur un site comportant de nombreuses informations. Lorsque le consommateur recherche des informations pour constituer son ensemble de considération, la présence d'un AVI, comme Lucie sur le site de SFR, peut l'aider à trouver l'information recherchée. Ensuite, dans la phase qui précède la vente, l'AVI peut pousser des produits auprès de prospects, par exemple, un crédit à la consommation. C'est par exemple le cas de Nicolas pour Finaref. Par ailleurs, il peut aussi jouer un rôle crucial en cas de problème rencontré au moment de l'achat sur un site de e-commerce (Tea de Discounteo). Un client peut, par exemple faire appel à l'agent s'il n'arrive pas à visualiser son panier. L'objectif est alors d'accroître le taux de 
conversion. Enfin, un agent peut intervenir après la vente pour répondre aux réclamations ou aux questions des clients concernant le suivi des commandes. C'est le cas de Clara, agent virtuel de Fnac.com, qui est capable de répondre aux questions relatives à l'état d'avancement de la commande grâce à son intégration avec le système d'information.

Dans un futur proche, les AVI seront capables de collecter des informations de contexte sur le prospect ou client en amont de la conversation (données de navigation, informations client...) afin de rendre l'agent davantage proactif, notamment dans les offres qu'il pourra faire, et d'améliorer la relation client. C'est déjà le cas avec Nicolas, l'AVI de Finaref qui est intégré à l'espace personnel du client, ce qui lui permet de proposer des produits additionnels aux personnes qui sont déjà clientes et identifiées grâce à la connexion de l'AVI au back office.

Le positionnement de l'agent sur le site doit être pensé en fonction de la mission qui lui est confiée. Par exemple, Tea est présente sur toutes les pages du site Discounteo.com, ce qui facilite les demandes d'information sur les produits, en phase de pré-vente. Clara, de fnac.com, n'est pas positionnée sur la page d'accueil ni sur les pages produits étant donné que sa mission se limite au service après-vente. En revanche, Lucie, ayant pour mission de guider les visiteurs lors de la navigation sur le site de SFR, est positionnée sur la page d'accueil. Sur le site de Finaref, Nicolas, dont une partie de la mission consiste à proposer des produits aux prospects, est positionné sur la page d'accueil.

\section{Tableau 1}

Les sites analysés et leurs agents virtuels

\begin{tabular}{|c|c|c|}
\hline Sites & Profil de l'AVI & Mission de l'AVI \\
\hline $\begin{array}{l}\text { SFR } \\
\text { (www.assistance.sfr.fr) } \\
\text { Opérateur de téléphonie } \\
\text { fixe, mobile et fournisseur } \\
\text { d'accès Internet. }\end{array}$ & $\begin{array}{l}\text { Lucie, mise en } \\
\text { place en juillet } \\
2009 \\
\text { Personnage animé } \\
\text { en 2D. }\end{array}$ & $\begin{array}{l}\text { Aide à la navigation au sein d'un site à } \\
\text { contenu riche. } \\
\text { Le centre de contact était surchargé de } \\
\text { demandes alors que les réponses étaient } \\
\text { disponibles sur le site. Le moteur classique } \\
\text { et les onglets ne suffisaient plus. } \\
\text { Lucie est capable de reconnaître la } \\
\text { navigation de l'internaute en temps réel } \\
\text { grâce à son intégration au système de } \\
\text { navigation: elle est proactive et peut } \\
\text { adapter son dialogue lors de chaque } \\
\text { conversation. }\end{array}$ \\
\hline Discounteo.com & Téa, mise en place & Aide à la vente \\
\hline
\end{tabular}




\begin{tabular}{|c|c|c|}
\hline Sites & Profil de l'AVI & Mission de l'AVI \\
\hline $\begin{array}{l}\text { Vente en liq } \\
\text { électroména } \\
\text { tech grand } \mathrm{p}\end{array}$ & $\begin{array}{l}\text { fin } 2005 \\
\text { Vidéo animée qui } \\
\text { représente une } \\
\text { hôtesse. }\end{array}$ & $\begin{array}{l}\text { Le site, créé en 2004, a connu une } \\
\text { croissance rapide. Dès 2005, Téa est } \\
\text { intégrée au site, avec pour mission de } \\
\text { répondre aux demandes des visiteurs, de } \\
\text { fournir une aide à la vente et un support } \\
\text { après-vente. Téa est directement connectée } \\
\text { au back office ce qui lui permet d'informer } \\
\text { directement le client du suivi de sa } \\
\text { commande. Téa est également reliée au } \\
\text { web analytic }\end{array}$ \\
\hline $\begin{array}{l}\text { Finaref } \\
\text { VAD de produits } \\
\text { financiers (crédits à la } \\
\text { consommation via les } \\
\text { enseignes de distribution). }\end{array}$ & $\begin{array}{l}\text { Nicolas } \\
\text { Boulanger, mis en } \\
\text { place fin } 2008 \\
\text { Photo } \\
\text { représentant un } \\
\text { conseiller typique } \\
\text { d'un établissement } \\
\text { financier. }\end{array}$ & $\begin{array}{l}\text { Conseil avant vente : Nicolas propose des } \\
\text { produits classiques aux prospects. } \\
\text { Fidélisation des clients : Nicolas est } \\
\text { intégré à l'espace personnel du client et au } \\
\text { back office. Il reconnaît le client et lui } \\
\text { propose des produits complémentaires } \\
\text { (prévoyance, assurance) en fonction de son } \\
\text { profil. }\end{array}$ \\
\hline $\begin{array}{l}\text { Fnac.com } \\
\text { Vente en liq } \\
\text { culturels et }\end{array}$ & $\begin{array}{l}\text { Clara, mise en } \\
\text { place fin } 2007 \\
\text { Vidéo animée } \\
\text { représentant une } \\
\text { conseillère typique } \\
\text { de la Fnac, grâce à } \\
\text { son uniforme. }\end{array}$ & $\begin{array}{l}\text { Aide au service après-vente } \\
\text { Les contacts clients liés au SAV étaient } \\
\text { trop nombreux ( } 710000 \text { contacts/an) et } \\
\text { augmentaient de } 20 \% \text { par an. } \\
\text { Clara est intégrée au back office ce qui lui } \\
\text { permet de renseigner le client à partir du } \\
\text { numéro de commande. Elle répond aux } \\
\text { questions relatives au suivi de commande, } \\
\text { aux pertes et retours, etc.). }\end{array}$ \\
\hline
\end{tabular}

\section{Des agents perfectibles}

Malgré les nombreuses améliorations dont ont pu bénéficier les agents virtuels, quelques faiblesses doivent être soulignées. La première concerne leur intelligence. Un test de pertinence des réponses apportées à quelques questions (encadré 3) montre qu'ils ont encore des progrès à faire avant de réussir le test de Turing (19). Le deuxième point sur lequel ils devraient faire l'objet d'améliorations porte sur leur capacité à se différencier en intégrant la voix, l'animation et/ou une apparence 3D.

\section{Encadré 3 - Test de pertinence des réponses}

Une vingtaine d'étudiants inscrits en deuxième année de Master ont joué le rôle du client mystère. Ils devaient engager une conversation avec l'un des AVI retenus pour l'étude, en respectant le scénario ci-dessous. Les conversations ont été sauvegardées et soumises à analyse. 
1) Poser une question en rapport avec la mission de l'AVI (recherche d'un produit ou service, demande concernant le suivi d'une commande, par exemple).

2) Poser une question pouvant introduire un contresens (par exemple «je suis à la recherche d'un aspirateur mais surtout pas un Dyson »).

3) Jouer un client qui perd patience parce que l'AVI n'a pas compris la question.

4) Poser deux questions personnelles : Quel âge avez-vous ? Etes-vous marié(e) ?

5) Poser des questions hors propos: Quel temps fait-il chez vous ? Voulez-vous prendre un verre avec moi ?

6) Demander à l'agent s'il parle anglais.

Afin de compléter cette étude, une conversation sur la base des questions ci-dessus a été engagée avec Anna, l'AVI d'Ikea. Il s'agit d'un personnage virtuel dessiné développé par la société Artificial Solutions qui fonctionne sur la base de mots-clés.

\section{- Une intelligence encore limitée...}

L'analyse des résultats obtenus lors du test de pertinence des réponses des différents agents sollicités peut être présentée autour de quatre points.

La capacité de l'agent à remplir sa mission. L'analyse des conversations montre que les AVI comprennent plus ou moins bien les questions en rapport direct avec leur mission. Par exemple, à la question «Combien coûte l'ADSL chez SFR ? », Lucie répond: «Apparemment, je n'ai pas bien compris votre problème. Peut-être qu'en reformulant votre question je pourrai vous aider ?». A la question : «Avez-vous des ordinateurs Apple ? », Téa, l'AVI de Discounteo renvoie vers les pages « Apple » mais ces pages sont vides car le site ne commercialise pas cette marque. Elle ne répond pas directement à la question. Avec Clara, l'AVI de la Fnac, il a fallu plusieurs échanges avant qu'elle demande les références de la commande. L'agent n'a pas répondu correctement à la question : «Bonjour, je viens de passer une commande. Quand seraije livré ? » mais a compris la question : «Où en est ma commande ? ». En revanche, à la question : «Bonjour, j'ai besoin d'un prêt pour acheter une voiture », Nicolas, l'AVI de Finaref, répond directement : «Je vous conseille le prêt personnel. Cliquez ici pour en savoir plus ». Le lien renvoie vers un formulaire de demande de prêt en ligne. L'échange est donc très rapide. Les AVI tombent parfois dans le piège des questions pouvant introduire des contresens. Par exemple, à la question «Finalement je voudrais un téléphone mobile mais pas un Nokia », Téa répond «Cliquez ici pour voir notre offre mobile ». En suivant le lien, on obtient toutes les marques, dont Nokia. Anna l'AVI d'Ikea, fonctionnant sur une base de mots-clés, reformule les propos de l'internaute et 
ouvre directement la page associée au mot-clé trouvé dans son langage naturel paramétré. Si dans la grande majorité des cas, les résultats proposés sont pertinents, des problèmes de compréhension se posent parfois. Par exemple, lorsqu'on lui demande « je souhaiterais acheter une armoire pour mon fils de 8 ans », Anna propose d'aller voir la sous-catégorie meubles de rangement enfant et ouvre une nouvelle fenêtre comportant les produits de cette catégorie. En revanche, lorsqu'on lui demande une armoire solide, elle propose (et ouvre la page correspondante) à la gamme d'armoires-penderies solitaires... soulignant ici la limite des AVI fonctionnant sur la base de mots-clés.

L'aptitude de l'agent à gérer les situations tendues. Lorsque le client mystère perd patience est met en cause la compétence de l'AVI «vous n'êtes pas vraiment intelligent », ou «vous êtes nul, vous ne comprenez rien à ce que je vous demande », invariablement, il réagit avec une expression du type : «Vous m’en voyez désolé... Je vais continuer à travailler pour être meilleur la prochaine fois. Je suis à votre écoute». En ce qui concerne l'AVI d'Ikea, le même type de réponse est proposé à ce type de remarque «désolée, mes programmateurs font de leur mieux pour améliorer mes connaissances. N'hésitez pas à reformuler votre question » ou «je suis désolée de ne pas toujours comprendre mais n'hésitez pas à reformuler votre question ou à me faire part de vos commentaires sur IKEA, ses produits et services ». On vérifie bien ici l'aptitude des AVI à encaisser les reproches et l'effort d'empathie, ce qui ne serait pas forcément le cas avec un conseiller, au téléphone.

\section{Le traitement des questions personnelles et hors propos et l'aptitude de l'agent à} recentrer la conversation sur sa mission. Les AVI répondent presque systématiquement aux questions personnelles (âge, statut marital), à l'exception de Lucie «voyons, on ne pose pas ce genre de questions à une femme ». Mais tous les agents que nous avons testés essaient de recentrer la conversation sur leur mission : « Mais arrêtons de parler de moi, je suis là pour vous aider ! ». Ils répondent également tous sur les questions hors propos telles que la météo, avec des formules toutes faites du type : «de mon bureau, j'aperçois un coin de ciel bleu ». Ils font parfois même de l'humour, lorsqu' on leur pose la question «voulez-vous prendre un verre ce soir ?», ils répondent quasiment tous : «J'évite de boire, ça me fait rouiller... ». En ce qui concerne l'AVI d'Ikea fonctionnant sur la base de mots-clés, les réponses proposées sont parfois inattendues. Par exemple, à la question «êtes-vous mariée ?» Elle répond «je vous 
informe que nous ne proposons pas le service Liste de Cadeaux. Néanmoins, je vous offre la possibilité de faire plaisir à vos proches, en leur offrant une carte cadeaux. Pour en savoir plus, je vous invite à consulter la page que je vous ouvre à l'instant (ouverture d'une nouvelle fenêtre sur les cartes cadeau Ikea). » De même, à la question «quel temps fait-il en Suède ? », elle répond «vous trouverez ici des informations sur les magasins IKEA dans le monde. »Enfin, si on lui propose de prendre un verre avec nous, elle répond « je vais vous montrer sur ce lien la sous-catégorie verre et carafes. Pour transporter vos verres plus facilement, nous mettons gratuitement à votre disposition des cartons que vous trouverez au rayon vaisselle. »

La maîtrise des langues étrangères par l'agent. Lorsqu'on s'adresse à eux en anglais, ils répondent soit «Je travaille actuellement sur la langue de Shakespeare mais je n'ai pas encore assez de bases pour dialoguer avec vous » soit « Ici, je parle français. Mais VirtuOz traite aussi l'espagnol, l'anglais, l'italien, le portugais, l'allemand, le hollandais et bientôt le chinois. » On peut s'interroger sur l'utilité de cette deuxième formulation. Il est peu probable que l'internaute moyen connaisse la société VirtuOz. Anna, quant à elle, propose un autre type de réponse plus approprié «I do speak English a little, like other interactive assistants in IKEA US or IKEA UK. To find out more about our services, we invite to check out the page that I'm opening at the moment » (ouverture de la page Services in English d'Ikea).

\section{- Une faible différenciation}

Les AVI intégrés aux sites Internet français sont encore faiblement différenciés à la fois en matière d'humanisation et d'apparence physique (encadré 4). Ils fonctionnent quasiment tous sur le principe d'une communication écrite. Or, il a été démontré que la voix est mieux perçue que les interactions basées uniquement sur l'écrit (6). Les automates audio ont un effet supérieur sur la confiance par rapport au texte écrit (16). Quant à leur apparence physique, ils sont quasiment tous en 2D, faiblement animés et ils ont un air de ressemblance. Cela peut s'expliquer par le fait que les acteurs sont en définitive peu nombreux, notamment en ce qui concerne l'enveloppe physique des agents. Par exemple, VirtuOz fait appel à Cantoche Production pour ses AVI. Pourtant, les progrès de la technologie ouvrent la voie à une plus grande différenciation des agents. Cette différenciation pourrait passer par la technologie 3D, l'animation et la 
customisation des AVI. On pourrait ainsi donner un plus grand choix à l'internaute quant à l'apparence de l'AVI. Souhaite-t-il communiquer avec un homme ou une femme, par exemple. Un synthétiseur de voix pourrait également être ajouté.

\section{Encadré 4 - Humanisation et anthropomorphisme}

«Le terme anthropomorphisme, dans un sens large, pris à la lettre de son étymologie, peut désigner l'acte de doter quelque chose de la forme humaine : créer de toutes pièces un objet ayant forme humaine au sens plastique du terme, ou revêtir un objet déjà existant de forme ou d'attributs humains » (Encyclopédie Universalis), alors que l'humanisation ou l'action d'humaniser «consiste à donner un caractère plus humain à quelque chose » (Dictionnaire Larousse). Ainsi, l'anthropomorphisme contient une dimension physique - donner une apparence humaine à un programme informatique, par exemple - alors que l'humanisation peut être obtenue en recourant à un ensemble d'éléments - comme un simple module conversationnel ou une messagerie instantanée - qui peuvent rendre le site Internet plus humain ou plus social. Un automate audio et un module conversationnel relèvent, par exemple, d'avantage de l'humanisation que de l'anthropomorphisme.

Les agents virtuels anthropomorphiques - ceux qui disposent d'une apparence humaine - font preuve d'un réalisme variable. Certains constituent une représentation fidèle de l'être humain (photographie / Nicolas Boulanger pour Finaref ; vidéo / Clara de la Fnac) ; d'autres ont une représentation digitale (Lucie pour SFR, Anna chez Ikea). Dans ce cas, l'AVI peut être animé ou non et être représenté en 2D ou 3D. La plupart des AVI intégrés aux sites marchands sont en 2D, faiblement animés. Le langage corporel de l'AVI - gestes, postures et regards - est peu développé.

Ce bilan critique portant sur les performances des AVI amène à s'interroger sur l'impact qu'ils peuvent avoir sur la relation client et sur le rôle qu'ils peuvent être amenés à jouer dans ce dispositif. Ce rôle doit être défini précisément, afin de contourner leurs faiblesses.

\section{Les apports des AVI en matière de relation client}

Comme cela a été souligné dans de précédentes recherches (2), la technologie des AVI peut contribuer à la mise en œuvre d'une politique de marketing relationnel (encadré 5).

\section{Encadré 5 - Du marketing relationnel à la gestion de la relation client}

L'intérêt croissant porté aux phénomènes relationnels en marketing a conduit, dans les années 1990, à l'émergence du marketing relationnel considéré par certains comme un nouveau paradigme et, par d'autres, comme une simple réaffirmation des principes du marketing (9). Le marketing relationnel désigne « toutes les activités marketing visant à établir, développer et maintenir de bonnes relations » (10). Le marketing relationnel à 
destination des clients est à la fois une politique et un ensemble d'outils destinés à établir des relations personnalisées, durables et satisfaisantes avec les clients, afin de gagner leur préférence et leur confiance à long terme. Pour établir et maintenir de bonnes relations avec les clients, il faut les connaître, leur parler, les écouter, reconnaître leur fidélité et si possible les associer à la vie de la marque. Cette nécessité permet de souligner le lien entre le marketing relationnel et la gestion de la relation client (ou CRM). En effet, le CRM consiste à rassembler des informations détaillées et individualisées sur les clients et à gérer avec soin tous les moments de contact avec eux.

L'amélioration de la relation peut s'analyser du point de vue du consommateur et de celui de l'entreprise. Du point de vue du consommateur, le caractère ludique de la conversation avec un interlocuteur virtuel favorise l'émergence d'un lien d'une nature différente, capable de générer de la confiance et de libérer la parole du consommateur. Du côté de l'entreprise, les AVI présentent de nombreux atouts notamment sur les plans de l'image, de la collecte et de l'exploitation immédiate d'informations sur les clients, de la qualité de navigation sur le site et en ce qui concerne la maîtrise des coûts opérationnels de la relation client. En effet, la gestion des contacts entrants est désormais intégrée et les entreprises peuvent orienter le client vers un canal approprié (email, téléphone, site Internet, agence ou magasin) en fonction du coût du contact selon le canal, de la valeur du client et/ou de la nature de sa demande. Certaines demandes d'informations sont répétitives et leur potentiel en matière de création de valeur pour l'entreprise est relativement limité alors que leur prise en charge, par un canal traditionnel - comme le téléphone et l'email - représente un coût important.

Il est proposé d'analyser ici les apports des AVI à la relation client selon plusieurs axes : leur influence sur les réponses des consommateurs, leur potentiel d'humanisation des sites et leur impact sur la qualité de service, leur intégration dans le dispositif multicanal de relation client et la maîtrise des coûts opérationnels de celle-ci.

\section{L’influence des AVI sur les réponses des consommateurs}

De nombreuses recherches ont souligné le rôle des AVI en matière de relation client. Selon certaines, la présence d'un AVI a un effet direct sur les réponses du consommateur qu'il s'agisse de confiance, d'intention d'achat, de recommandation (8) ou du pouvoir de rétention des sites (stikiness), défini dans ce contexte comme la capacité du site à créer une relation forte et durable avec un visiteur ou client (1). L'effet de l'AVI sur les réponses affectives et comportementales du consommateur 
n'est corroboré que pour la dimension comportementale du pouvoir de rétention temps passé sur le site et nombre de pages vues - mais pas pour la dimension intentionnelle - intention de revisiter et de recommander le site (1).

Par ailleurs, les effets des AVI sur certaines variables (plaisir, éveil, flow, confiance, valeurs hédoniques/utilitaires, attitude à l'égard du site, comportement futur...) sont médiatisés soit par la présence sociale, soit par la crédibilité et les réponses émotionnelles $(3,11,18)$. Enfin, certaines caractéristiques du consommateur, comme son implication, modèrent l'effet de persuasion d'un AVI. Un AVI physiquement attractif est plus persuasif auprès des internautes peu impliqués alors que chez les consommateurs impliqués, c'est seulement lorsqu'il est perçu comme plus crédible que les effets sur la satisfaction, l'attitude à l'égard du produit et l'intention d'achat sont les plus forts (4).

\section{L'humanisation du site}

D'un point de vue plus qualitatif, la présence d'un AVI sur un site Internet peut aider à enrichir l'expérience client. En effet, les AVI disposent d'une apparence humaine et deviennent plus sociables du fait qu'ils sont capables de tenir une conversation avec un être humain. Cela contribue à humaniser le site et la relation client, à améliorer la présence sociale perçue (encadré 6) et à favoriser la création d'un lien social entre le site et le consommateur (14).

Celui-ci, bien qu'il ne soit pas dupe, peut imaginer qu'il est en relation directe avec un assistant clientèle. Des clients de VirtuOz ont, d'ailleurs relaté des tentatives de « drague » de leurs agents et des pics de conversations nocturnes...

\section{Encadré 6 - La présence sociale}

Les chercheurs se sont depuis longtemps intéressés aux dispositifs qui permettent aux utilisateurs d'imaginer qu'ils sont en interaction avec d'autres humains, à défaut de l'être réellement. Le concept de présence sociale peut se définir comme le sentiment d'être en présence d'autres personnes lorsque la communication n'a pas lieu en face-àface mais est intermédiée par un moyen technologique (17).

Plus récemment, avec la montée en puissance d'Internet, les chercheurs ont pris conscience que les facteurs liés à la facilité d'utilisation et à l'utilité de la technologie n'étaient pas suffisants pour comprendre la complexité de la relation entre l'utilisateur (l'internaute) et l'ordinateur (le site Internet). Un nouveau paradigme a alors vu le jour ; il s'agit du paradigme CASA pour Conputers Are Social Actors (13). Le concept de 
présence sociale, initialement développé dans un contexte de communication, a trouvé une nouvelle application dans la relation entre l'utilisateur et l'ordinateur. De nombreuses recherches montrent que les individus attribuent des caractéristiques humaines aux ordinateurs et appliquent les mêmes règles sociales et les mêmes attentes lorsqu'ils interagissent avec ceux-ci. Les règles de politesse, les stéréotypes liés au genre, la personnalité et les effets de flatterie s'appliquent également entre l'homme et l'ordinateur (12).

Il y a deux moyens d'instiller un sentiment de présence sociale sur un site web (3) : soit fournir des moyens qui permettent une réelle interaction avec d'autres humains (email, chat, forum, communautés virtuelles, assistant clientèle), soit stimuler l'imagination afin de donner l'impression d'une interaction avec d'autres personnes (par des photos ou des vidéos dont le contenu est socialement riche, par du texte socialement riche, des salutations personnalisées, par la parole, par des AVI). Dans le premier cas, ces interfaces de communication peuvent être synchrones (ils permettent une communication instantanée) ou asynchrones (les réponses sont différées) mais la réponse doit être générée par un humain. Dans le cas d'une interaction imaginaire, en revanche, la réponse est générée automatiquement par un ordinateur. Les agents virtuels ont donc pour objectif de créer un sentiment de présence sociale en contribuant à améliorer l'atmosphère du site Internet (8).

\section{L'amélioration de la qualité du service}

En contribuant à ré-humaniser la relation entre l'internaute et le site web, l'AVI peut participer à l'amélioration de la qualité perçue de l'interaction en ligne. L'AVI étant sensé remplacer le personnel, dans certaines situations, il est intéressant de s'interroger sur l'impact qu'il peut avoir sur les cinq dimensions de la qualité de service traditionnelle, c'est-à-dire pour un service interpersonnel (15). Le tableau ci-dessous (tableau 2) présente les contributions des AVI à la qualité de service traditionnelle et montre quels sont leurs atouts éventuels par rapport à un agent humain.

\section{Tableau 2}

\section{Contribution des AVI à la qualité de service}

\begin{tabular}{|l|l|}
\hline \multicolumn{1}{|c|}{$\begin{array}{c}\text { Dimensions de la qualité } \\
\text { de service }\end{array}$} & \multicolumn{1}{c|}{$\begin{array}{c}\text { Avantages de l'agent virtuel } \\
\text { par rapport à un agent humain }\end{array}$} \\
\hline $\begin{array}{l}\text { Tangibilité } \\
\text { Eléments matériels tels que } \\
\text { l'apparence physique des } \\
\text { locaux, des équipements, du } \\
\text { personnel et des documents. }\end{array}$ & $\begin{array}{l}\text { L'agent virtuel peut être assimilé au personnel en contact avec } \\
\text { les clients (Clara, de la Fnac, est habillée comme un vendeur } \\
\text { Fnac). Il donne une apparence humaine au support client. En } \\
\text { outre, les AVI sont, en règle générale, plutôt physiquement } \\
\text { attractifs, qu'ils soient représentés par une vidéo ou par une } \\
\text { image 2D. Or, en communication, on sait de longue date, } \\
\text { qu'une personne attractive est davantage persuasive. }\end{array}$ \\
\hline $\begin{array}{l}\text { Fiabilité } \\
\text { Capacité à réaliser le service } \\
\text { de manière sûre et précise. }\end{array}$ & $\begin{array}{l}\text { La fiabilité de l'AVI s'apprécie à partir du nombre de } \\
\text { conversations réussies. Dans des circonstances identiques, } \\
\text { l'AVI fournit toujours la même réponse. Il n'y a pas de }\end{array}$ \\
\hline
\end{tabular}




\begin{tabular}{|c|c|}
\hline $\begin{array}{c}\text { Dimensions de la qualité } \\
\text { de service }\end{array}$ & $\begin{array}{c}\text { Avantages de l'agent virtuel } \\
\text { par rapport à un agent humain }\end{array}$ \\
\hline & subjectivité dans l'appréciation de la situation. \\
\hline $\begin{array}{l}\text { Réactivité } \\
\text { Volonté d'aider le client en } \\
\text { lui fournissant un service } \\
\text { rapide et adapté. }\end{array}$ & $\begin{array}{l}\text { L'agent virtuel n'a pas d'autre mission que d'apporter son aide } \\
\text { au client. Il fournit une réponse immédiate. Il n'y a pas de file } \\
\text { d'attente. Il peut traiter jusqu'à } 4500 \text { demandes en même } \\
\text { temps. }\end{array}$ \\
\hline $\begin{array}{l}\text { Réassurance } \\
\text { Capacité à inspirer } \\
\text { confiance. Elle se fonde sur } \\
\text { la compétence, la crédibilité, } \\
\text { la sécurité et la courtoisie. }\end{array}$ & $\begin{array}{l}\text { La compétence de l'agent virtuel est cruciale pour rassurer le } \\
\text { client. Pour cela, il ne doit pas systématiquement renvoyer vers } \\
\text { un autre canal. Dans une expérience menée en } 2005,52 \% \text { des } \\
\text { personnes jugeaient l'agent virtuel davantage digne de } \\
\text { confiance que le vendeur en magasin, pour } 41 \% \text { c'était } \\
\text { l'inverse et } 7 \% \text { ne voyaient pas de différence (7). }\end{array}$ \\
\hline $\begin{array}{l}\text { Empathie } \\
\text { Accorder une attention } \\
\text { individualisée au client, faire } \\
\text { preuve de compréhension à } \\
\text { son égard. }\end{array}$ & $\begin{array}{l}\text { Le sentiment d'être en interaction avec un semblable et } \\
\text { l'humeur constante de l'agent peuvent créer une impression } \\
\text { d'empathie. Le fait que l'AVI n'emploie pas de jargon } \\
\text { technique est également susceptible d'accroître l'impression } \\
\text { d'empathie. Une personne humaine pourrait être tentée de le } \\
\text { faire pour se « débarrasser » d'un client mécontent. L'aptitude } \\
\text { potentielle des AVI à maîtriser des langues étrangères peut } \\
\text { également contribuer à améliorer l'empathie. }\end{array}$ \\
\hline
\end{tabular}

Cette analyse montre que les AVI sont susceptibles d'améliorer la plupart des dimensions de la qualité de service. La seule dimension pour laquelle, à l'heure actuelle, cet effet serait plus mitigé est la réassurance car, comme le montre le test de pertinence, ils ne sont pas encore capables de traiter l'ensemble des demandes, y compris lorsqu'elles relèvent de leur mission.

\section{L'intégration des AVI dans le dispositif multicanal de relation client}

Les AVI constituent un maillon important dans le dispositif multicanal des sites Internet qu'il s'agisse de pure players ou de click \& mortar. Les différents canaux de contact de la relation client peuvent être positionnés selon deux axes. Le premier oppose le web self-service à l'assistance directe par l'entreprise. Le second oppose les canaux synchrones et les canaux asynchrones (figure 1). Selon ce schéma, l'AVI est un dispositif de communication synchrone en self-service. Il constitue un point d'entrée de la relation client en ce sens qu'il peut renvoyer un internaute vers les autres canaux de contact alors qu'aucun canal ne va renvoyer vers l'AVI. Dans le cas d'un basculement vers un autre canal, il semble préférable d'opter pour un canal synchrone (téléphone, messagerie instantanée, click-to-call) plutôt qu'asynchrone (email, et SMS) car le fait de 
passer d'un canal instantané à un canal différé pourrait créer un sentiment d'insatisfaction chez le client ou prospect.

\section{Figure 1}

\section{Place des AVI dans la stratégie multicanal de relation client}

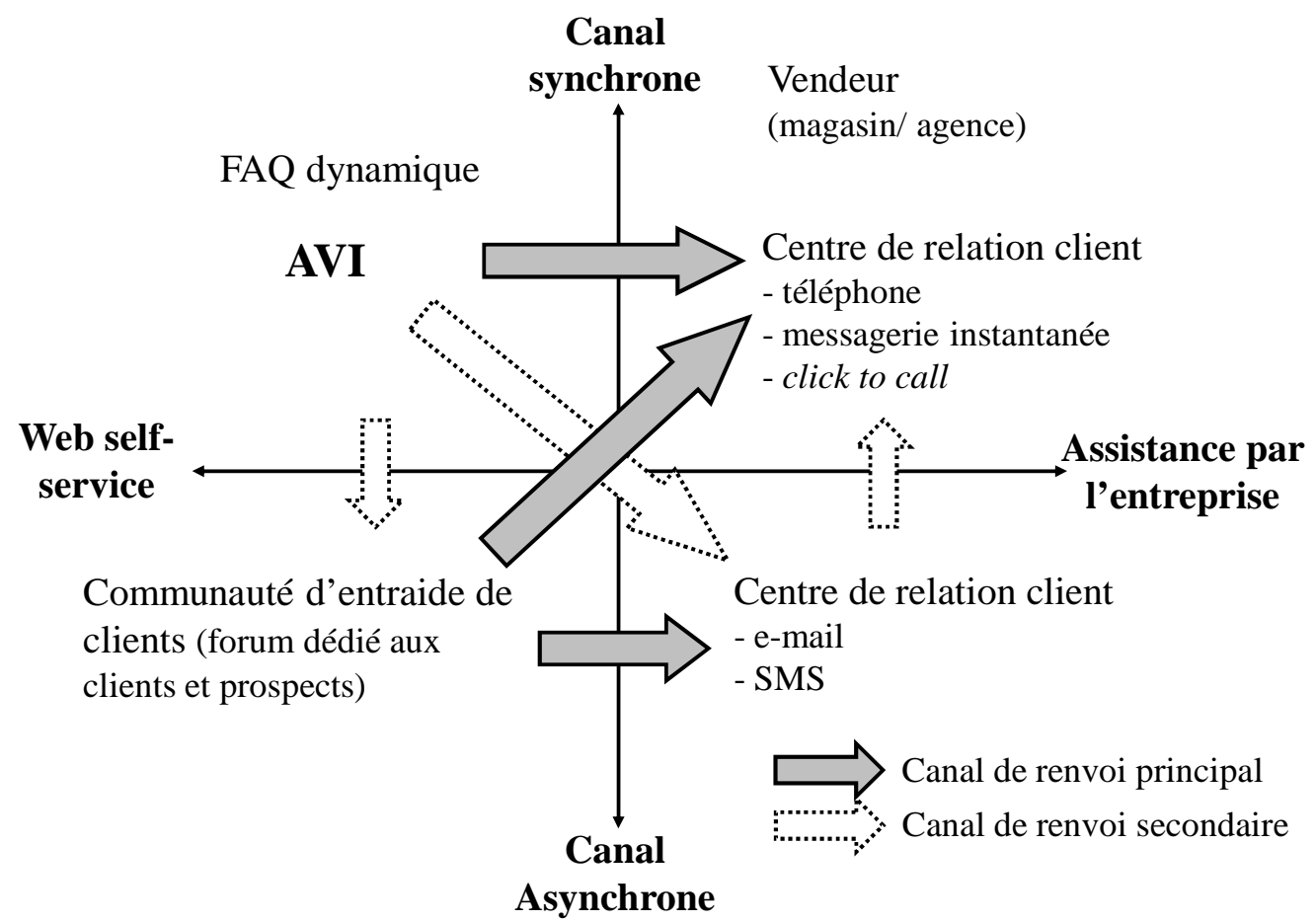

Par rapport aux dispositifs qui relèvent du web 2.0, comme par exemple les communautés de clients, parmi lesquelles La Fourmilière de Cdiscount, l'AVI présente plusieurs avantages. Il fournit systématiquement des réponses aux questions posées alors, qu'au sein d'une communauté, celles-ci reposent sur la bienveillance des membres. La communication avec un AVI est synchrone alors que les communautés fonctionnent en général sur le modèle d'un forum, canal asynchrone. En revanche, il se peut que les réponses obtenues au sein d'un forum soient plus pertinentes sur des questions spécifiques. Cela est d'autant plus probable pour les communautés qui disposent d'un community manager.

\section{La maîtrise des coûts opérationnels de la relation client}


Les AVI relèvent de la logique du web self-service. Celle-ci présente un intérêt pour l'entreprise car le coût d'un contact par des moyens de communications plus directs comme l'email et le téléphone reste élevé. Les AVI sont, en effet, particulièrement utiles pour améliorer la productivité et traiter, à faible coût, des requêtes répétitives. Lorsqu'il s'agit de demandes d'information, ils se substituent aisément aux FAQ et autres aides à l'orientation (moteurs de recherche internes, onglets...). Selon VirtuOz, de 5 à $25 \%$ des visiteurs s'adressent à un AVI, en fonction de son positionnement sur le site.

La performance associée à un agent virtuel ne peut être envisagée sans prendre en compte la mission et les objectifs qui lui sont assignés. En effet, les indicateurs de performance dépendront des objectifs recherchés qui, eux mêmes, dépendent de la mission confiée à l'agent. D'après VirtuOz, les AVI se voient confier quatre types de missions - aide à la navigation, conseil avant-vente, aide à l'achat et SAV -. Or, l'analyse des missions des quatre agents étudiés révèle qu'elles sont en réalité plus nombreuses. Nous en avons identifié six : aide à la navigation, conseil avant-vente, aide au processus d'achat, fidélisation, aide au SAV et connaissance client. Afin de mieux comprendre les enjeux des AVI en matière de maîtrise des coûts opérationnels de la relation client, nous avons tenté d'associer à chacune des missions des AVI des objectifs spécifiques et des indicateurs de performance (tableau 3).

\section{Tableau 3}

La mesure de la performance en fonction des missions de l'AVI

\begin{tabular}{|c|c|c|}
\hline Mission & Objectifs & Indicateurs de performance \\
\hline $\begin{array}{l}\text { Aide à la } \\
\text { navigation } \\
\text { Faciliter l'accès à } \\
\text { l'information } \\
\text { pour les } \\
\text { riches sites } \\
\text { contenu. }\end{array}$ & $\begin{array}{l}\text { Réduire le flux de contacts } \\
\text { entrants via les autres canaux. } \\
\text { Apporter des réponses } \\
\text { pertinentes aux clients et } \\
\text { prospects. } \\
\text { Augmenter la satisfaction des } \\
\text { visiteurs. }\end{array}$ & $\begin{array}{l}\text { Evolution du nombre de contacts } \\
\text { entrants. } \\
\text { Optimisation du taux de réponse. } \\
\text { Taux de satisfaction. } \\
25 \% \text { des visiteurs du site SFR } \\
\text { dialoguent avec Lucie et } 86 \% \text { d'entre } \\
\text { eux sont satisfaits par les réponses } \\
\text { obtenues. }\end{array}$ \\
\hline 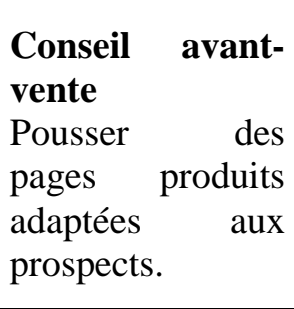 & $\begin{array}{l}\text { Recruter de nouveaux clients } \\
\text { Augmenter le trafic vers } \\
\text { certaines pages. }\end{array}$ & $\begin{array}{l}\text { Clients recrutés grâce à l'AVI. } \\
\text { Trafic vers les pages commerciales. } \\
\text { Temps passé sur les pages produits. } \\
\text { Nombre de pages vues. } \\
\text { Sur le site Discounteo le temps par } \\
\text { page est multiplié par } 4 \text { et le nombre de } \\
\text { pages vues par 2,5. }\end{array}$ \\
\hline $\begin{array}{l}\text { Aide } \\
\text { processus }\end{array}$ & $\begin{array}{l}\text { Réduire le nombre d'achats } \\
\text { abandonnés. }\end{array}$ & $\begin{array}{l}\text { Taux de transformation. } \\
\text { Nombre d'achats abandonnés. }\end{array}$ \\
\hline
\end{tabular}




\begin{tabular}{|c|c|c|}
\hline Mission & Objectifs & Indicateurs de performance \\
\hline $\begin{array}{l}\text { d'achat } \\
\text { Assister le client, } \\
\text { lors de l'achat, } \\
\text { pour éviter de le } \\
\text { perdre. }\end{array}$ & $\begin{array}{l}\text { Augmenter le chiffre } \\
\text { d'affaires. } \\
\text { Accroître le panier moyen. } \\
\text { Accroître le temps passé sur le } \\
\text { site. }\end{array}$ & $\begin{array}{l}\text { Panier moyen. } \\
\text { Sur le site Discounteo, le taux de } \\
\text { transformation a été multiplié par } 2 \text { et } \\
\text { le panier moyen a augmenté de } 30 \% \text {. }\end{array}$ \\
\hline 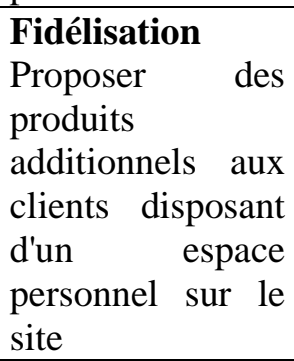 & $\begin{array}{l}\text { Inciter le client à acheter } \\
\text { d'autres produits (cross } \\
\text { selling) ou à monter en gamme } \\
\text { (up selling). } \\
\text { Augmenter le panier moyen. }\end{array}$ & $\begin{array}{l}\text { Taux de conversion. } \\
\text { Nombre de produits additionnels } \\
\text { placés. } \\
\text { Panier moyen. } \\
\begin{array}{l}\text { Emma apporte } 100 \text { à } 200 \text { ventes } \\
\text { additionnelles par jour à MMA. }\end{array}\end{array}$ \\
\hline $\begin{array}{lr}\text { Aide au SAV } & \\
\text { Suivi } & \text { de } \\
\text { commande } & \text { et } \\
\text { gestion } & \text { des } \\
\text { réclamations. } & \\
\end{array}$ & $\begin{array}{l}\text { Réduire le flux de contacts } \\
\text { entrants via les autres canaux. } \\
\text { Faire baisser les coûts du } \\
\text { service client. }\end{array}$ & $\begin{array}{l}\text { Nombre de contacts entrants. } \\
\text { Evolution des coûts opérationnels. } \\
\text { Sur Fnac.com, l'AVI a permis de } \\
\text { réduite de } 40 \% \text { le nombre d'emails et } \\
\text { de } 27 \% \text { les coûts opérationnels. }\end{array}$ \\
\hline $\begin{array}{l}\text { Connaissance du } \\
\text { client } \\
\text { Capturer la voix } \\
\text { du client ou du } \\
\text { prospect: } \\
\text { collecter, } \\
\text { enregistrer et } \\
\text { analyser les } \\
\text { conversations. }\end{array}$ & $\begin{array}{l}\text { Favoriser les réclamations en } \\
\text { incitant les silent sufferers à le } \\
\text { faire }^{6} \text {. } \\
\text { Identifier les mots-clés utilisés } \\
\text { par les clients ou prospects. } \\
\text { Augmenter les performances } \\
\text { de l'agent. } \\
\text { Accroître la réactivité des } \\
\text { centres de contact. } \\
\text { Identifier les causes } \\
\text { d'insatisfaction et les besoins } \\
\text { des clients ou prospects. }\end{array}$ & $\begin{array}{l}\text { Taux de satisfaction. } \\
\text { Taux de conversations réussies. } \\
\text { Productivité des centres de contact. } \\
\text { Sur Voyages-sncf.com, l'interaction } \\
\text { avec Léa a permis de faire remonter les } \\
\text { questions liées aux déplacements avec } \\
\text { des animaux de compagnie entraînant } \\
\text { une clarification de ces informations } \\
\text { sur le site. } \\
\text { Sur Fnac.com, l'interaction avec Clara } \\
\text { se termine par une enquête de } \\
\text { satisfaction (Net Promoter Score). }\end{array}$ \\
\hline
\end{tabular}

\section{Conclusion}

Aujourd'hui, les agents virtuels son capables de dialoguer avec les internautes en langage naturel, de façon personnalisée, avec des réponses plus ou moins pertinentes, tout en s'intégrant dans le dispositif multicanal de relation client de l'entreprise. Nous avons souligné dans cet article un certain nombre d'avantages caractérisant les assistants virtuels mais ils ne sont pas pour autant exempts de limites. En effet, le recours à un AVI présente des limites, parmi lesquelles, celles liées à l'intelligence artificielle : risque de conflit entre agents virtuels et collaborateurs humains employés par le support client, risque d'une indisponibilité temporaire de l'AVI, aversion de certaines personnes à l'égard de la technologie.

Ainsi, la plupart des sites disposant d'un AVI contiennent un dispositif qui permet d'établir un contact direct avec un assistant humain lorsque l'agent virtuel n'est pas en 
mesure de donner une réponse satisfaisante au client. Même s'ils se trouvent en amont de tous les canaux, les AVI sont intégrés dans la stratégie multicanal de relation client des entreprises. C'est le cas sur le site Paypal. Un prospect qui n'arrive pas à activer le compte qu'il vient de créer, est redirigé vers un numéro de téléphone lorsque l'AVI n'est pas en mesure de débloquer la situation. La mise en place d'un AVI correspond, en définitive, à l'automatisation et à la spécialisation de certaines tâches liées à la gestion de la relation client. D'un point de vue pratique, pour les gestionnaires de sites, il convient de positionner l'AVI comme point d'entrée de la relation client et de bien définir son rôle. Celui-ci peut consister à filtrer les contacts, donner des réponses de premier niveau et, lorsque la question dépasse ses compétences, à passer la main à un conseiller humain, celui-ci ayant à sa disposition toutes les informations déjà échangées. Le client ou prospect bénéficie ainsi d'une réponse immédiate. Il n'est pas obligé de solliciter un autre canal. Le rôle de l'humain est valorisé : il intervient en tant qu'expert et non plus pour traiter des demandes récurrentes. Ainsi, eBay Australie a pu noter une augmentation de la productivité des agents humains grâce au rôle de pré-qualification des demandes par l'AVI. Par ailleurs, selon VituOz, moins de $10 \%$ des conversations commencées avec un agent virtuel montent en escalade et se terminent, dans la même fenêtre, avec un agent humain sous forme de live chat. La manière dont l'agent humain a traité la problématique client est ensuite analysée pour améliorer l'agent virtuel en conséquence. Il est donc utopique de penser que les AVI puissent un jour prendre la place des conseillers humains. L'AVI contribue à renforcer les équipes et permet aux conseillers de se consacrer aux demandes clients à forte valeur ajoutée ou aux problématiques complexes. Lors de leur mise en place, il faut, également, une période d'apprentissage, qui correspond à un entraînement privé de l'agent, avant qu'il ne soit parfaitement opérationnel. Cet apprentissage s'effectue, pour partie, avec le personnel des plateformes de relation client auxquelles il va être intégré.

Par ailleurs, il ne faut pas oublier que l'un des objectifs du web self-service, en général, et des AVI, en particulier, est une meilleure maîtrise des coûts opérationnels associés à la relation client. L'un des postes que souhaitent réduire les responsables CRM est celui des charges salariales. La mise en place d'un AVI pourrait donc être ressentie comme une menace sérieuse de la part des personnels associés au support client, qu'il s'agisse d'une plateforme téléphonique ou d'une plateforme de contact plus globale qui gère 
également les emails entrants. D'un point de vue managérial, l'AVI peut être présenté au personnel des centres de relation client comme un moyen de maintenir les plateformes en France, en raison de l'allègement de leurs coûts de fonctionnement, et d'améliorer la qualité de leur travail. En effet, le personnel pourra se focaliser sur les demandes moins récurrentes et plus complexes, nécessitant une réelle expertise. L'enjeu est donc, pour l'entreprise, de bien répartir les demandes des clients entre traitement virtuel automatisé et traitement humain afin de proposer la meilleure expérience utilisateur tout en optimisant ses ressources.

Les gestionnaires de sites doivent également s'interroger sur les conséquences de l'indisponibilité temporaire de l'AVI. Que se passerait-il au niveau du dispositif de relation client? Les autres canaux de contact seraient-ils capables, techniquement, de prendre le relais? Une telle panne aurait des effets dommageables en matière d'image mais se traduirait probablement aussi par un manque à gagner si l'AVI se voyait confier une mission d'assistant à la vente. En revanche, l'indisponibilité d'un AVI, en raison d'une sollicitation trop importante, ne semble pas à l'ordre du jour, selon VirtuOz.

Enfin, il ne faut pas sous-estimer l'aversion de certaines personnes à l'égard de la technologie et, comme le souligne une récente étude ${ }^{7}$, il n'est pas certain que les technophobes disparaîtront avec les nouvelles générations. Les personnes habituées à un contact direct par téléphone ou en face-à-face pourraient montrer une faible propension à recourir aux AVI. Il est donc important de laisser le client choisir le mode de contact qui lui convient. Imposer un canal pourrait en effet entraîner une dégradation de la qualité perçue de la relation.

\section{Notes}

1 Agent virtuel intelligent : simple gadget ou véritable outil de relation client ? www.webmarketing.com, 4 janvier 2010.

2 Diane Clarkson, It's time to give virtual agents another look, Forrester, 18 décembre 2009.

3 Propos tenus par Laurent Landowski, l'un des fondateurs de VirtuOz, interviewé par Flore Fauconnier, JDN « Nos AVI ne cessent jamais de monter en intelligence », 2 décembre 2009.

4 Selon VirtuOz, les coûts standards de développement et d'intégration d'un AVI à un site s'évaluent à 3 mois de journée homme (VirtuOz ne souhaite pas communiquer de budget précis, chaque projet étant différent). Si le projet est plus complexe (interfaçage avec le back-office, gestion multicanal...), des coûts additionnels sont à prévoir en fonction de l'ampleur dudit projet. 
5 Baromètre 2010 de la relation client, Les Echos, «Les services clients dopés par la crise », 21 janvier 2010, p. 10.

6 Les silent sufferers sont des internautes qui ne trouvent pas de réponses à leurs questions et qui ne prennent pas la peine de contacter le service client par email ou par téléphone. Insatisfaits, ils quittent « silencieusement » le site web et n'y retourneront probablement pas.

7 « Nouveaux comportements et nouvelles attentes des consommateurs en matière de relation client », http://blogs.orange-business.com/live-france/2011/02/vers-la-fin-du-modele-multicanal-en-libre-service.html (28/03/2011)

\section{Références}

(1) Diesbach B. B., Chandon J. L. et Galan J.P. (2007), Effets de la présence et de la congruence d'un agent virtuel incarné sur le pouvoir de rétention du site web, Actes $d u$ $23^{\text {ième }}$ Congrès International de l'Association Française du Marketing, 31 mai- ${ }^{\mathrm{er}}$ juin 2007, Aix-les-Bains.

(2) Gauzente C. et Guilloux V. (2003), Marketing relationnel sur Internet : quelle place

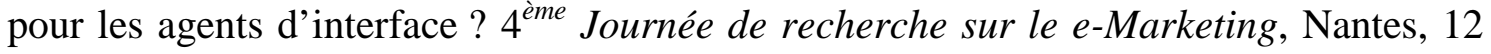
septembre.

(3) Hassanein K. et Head M. (2007), Manipulating perceived social presence through the web interface and its impact on attitude towards online shopping, International Journal of Human-Computer studies, 689-708.

(4) Holzwarth M., Janiszewski C. et Neumann M. (2006), The influence of Avatars on online consumer shopping behavior. Journal of Marketing, 70 (October 2006), 19-36.

(5) Huang S.-I. et Lin F.-R. (2007), The design and evaluation of an intelligent sales agent (ISA) for online persuasion and negotiation, Electronic Commerce Research and Applications, 6, 285-296.

(6) Jensen C., Farnham S., Drucker S. et Kollock P. (2000), The effect of communication modalidy on cooperation in online environment, In Proceedings of the 2000 conference on Human factors in computing systems, 470-477, New York, ACM Press.

(7) Komiak S. Y., Wang W. et Benbasat I. (2005), Trust building in virtual salespersons versus in human salespersons: similarities and differences, e-Service Journal, 3, 3,4963.

(8) Lemoine J.-F. et Notebaert J.-F. (2009), L'influence des agents virtuels sur la confiance des internautes vis-à-vis d'un site web, Communications of the IBIMA, $11^{\text {th }}$ IBIMA Conference, 4-6 janvier, Le Caire.

(9) Marion G. (2001), Le marketing relationnel existe-t-il ?, Décisions Marketing, 22, Janvier-Avril, 7-16.

(10) Morgan R.M. et Hunt S.D. (1994), The commitment-trust theory of relationship marketing, Journal of Marketing, 58, juillet, 20-38.

(11) Nan N., Anghelcev G., Myers J., Sar S. et Faber R. (2006), What if a web site can talk? Exploring the persuasive effects of web-based anthropomorphic agents, Journalism and Mass Communication Quarterly, 83, 3, 615-631.

(12) Nass C. et Moon Y. (2000), Machines and mindlessness: social responses to computers, Journal of Social issues, 56, 1, 81-103. 
(13) Nass C., Moon Y., Fogg B., Reeves B. et Dryer D. (1995), Can computer personalities be human personalities ? International Journal of Human-Computer Studies, 43, 223-239.

(14) Notebaert J.-F. (2005), L'interface homme-machine en commerce électronique : vers une création de lien social comme outil de positionnement stratégique, Revue Française du Marketing, 205, 5, 71-90.

(15) Parasuraman A., Zeithaml V., et Berry L. (1988), SERVQUAL: a multiple item scale for measuring consumer perception s of Service Quality, Journal of Retailing, 64, $1,12-40$.

(16) Qiu L. et Benbasat I. (2005), Online consumer trust and live help interfaces: the effects of Text-To-Speech Voice and Three-Dimensional Avatars, International Journal of Human-Computer Interaction, 19, 1, 75-94.

(17) Short J., Williams E. et Christie B. (1976), The social psychology of telecommunication, London Wiley.

(18) Wang L. C., Baker J. Wagner J. A. et Wakefield K. (2007), Can a retail website be social? Journal of Marketing, 71, July, 143-157.

(19) Turing A. M. (1950), Computing machinery and intelligence, Mind, 59, 36, 433460. 